\title{
Changes in the spatial organization of the uterine vasculature during implantation in the rat
}

\author{
P. A. W. Rogers, C. R. Murphy and B. J. Gannon \\ Department of Human Morphology, School of Medicine, Flinders University of South Australia, \\ Bedford Park, South Australia 5042
}

\begin{abstract}
Summary. The vascular corrosion casting/scanning electron microscope technique was used to investigate differential uterine elongation at the time of implantation in the rat. Casts were made on Days 4,5 and 6 of pregnancy (Day $1=$ the day of finding spermatozoa in the vaginal smear), implantation sites being identified by an i.v. injection of Evans' blue before casting. By Day 6 the endometrial blood vessel network within each implantation site was widely spaced compared with that in other regions. We suggest that blastocyst spacing is achieved, in part, by differential uterine elongation at the implantation sites, apparently as a result of tissue hydration subsequent to increased vascular permeability.
\end{abstract}

\section{Introduction}

In polytocous species implanting blastocysts are distributed more or less evenly along the length of the uterus (Finn \& Porter, 1975). However, the mechanism responsible for this spacing is not well understood. Mossman (1937) suggested that the first blastocyst to implant was the one closest to the utero-tubal junction and that it and each succeeding blastocyst produced around itself a refractory zone in which other blastocysts could not implant. Reynolds (1949), quoting the observations of Hammond (1935), pointed out that implantation stimulates growth of the surrounding parts of the uterus which would increase the distance between blastocysts and so improve spacing. Similar observations have been made by Perry \& Rowlands (1962) on the pig and by Finn (1968) on the mouse. Boving (1954) and McLaren \& Michie (1959) postulated that myometrial activity was responsible for the first general spacing of the blastocysts in the rat uterus; further evidence for this theory came from the work of Pusey, Kelly, Bradshaw \& Porter (1980).

The present study employed the corrosion vascular casting/scanning electron microscope method (Murakami, 1971; Gannon, 1978) to determine whether changes in uterine blood vessel organization and spatial distribution during the early stages of implantation provide insight into how implanting blastocysts are spaced in the rat.

\section{Materials and Methods}

Female Porton rats $(166-289 \mathrm{~g})$ in pro-oestrus at $16: 00 \mathrm{~h}$ were caged overnight with a male. Vaginal smears were taken the next morning at 09:00 h; the appearance of spermatozoa in a smear was considered as evidence of mating. The day of finding a positive vaginal smear was therefore Day 1 of pregnancy. Casts were made of the vasculature of 20 females, 4 between 11:00 and 12:45 h on Day 4 of pregnancy, 9 between $11: 30$ and 16:30 h on Day 5, and 7 between 11:30 and 15:00 h on Day 6. At $15 \mathrm{~min}$ before casting $1 \mathrm{ml}$ of 1\% Evans' blue in saline 
$(9 \mathrm{~g} \mathrm{NaCl} / 1)$ was injected into each animal via the tail vein. The details of the vascular casting technique are described by Rogers \& Gannon (1981). Briefly, the posterior half of the vasculature was flushed, via a cannula tied into the descending thoracic aorta, with a $37^{\circ} \mathrm{C}$ solution of $0.9 \%(\mathrm{w} / \mathrm{v}) \mathrm{NaCl}$ containing polyvinylpyrrolidone (mol. wt 40 000: Sigma, St Louis, Missouri, U.S.A.) at $58.7 \mathrm{~g} / \mathrm{l}, 10 \mathrm{i} . \mathrm{u}$. heparin (Glaxo, Melbourne) $/ \mathrm{ml}$ and $1 \mu \mathrm{g}$ papaverine (Bull Laboratories, Melbourne)/ml. This was followed by the casting medium which consisted of: 20 ml methyl methacrylate (Polysciences, Warrington, Pennsylvania, U.S.A.), prepolymerized to a viscosity of $2 \cdot 7-4 \cdot 0$ cst ( 1 stoke $=10^{-4} \mathrm{~m}^{2} / \mathrm{sec}$ ), $7 \mathrm{ml}$ hydroxypropyl methacrylate (Polaron, Watford, U.K.), $250 \mathrm{~g}$ benzoyl peroxide (Polysciences), and $0.4 \mathrm{ml} n, n$-dimethyl aniline (Merck, Munich, West Germany). The first $85 \%$ of the casting medium was infused at a pressure of 90 $\mathrm{mmHg}$ (measured in the abdominal aorta at the level of the kidneys), while the last $15 \%$ was infused at a pressure of $20 \mathrm{mmHg}$ with the venous out-flow cut in the vena cava clamped shut; this left the casting medium in the vascular system under a slight positive pressure while it polymerized. Casts were left to harden overnight in warm water $\left(45-50^{\circ} \mathrm{C}\right)$, and the uterus was dissected out the following morning. Implantation sites were identified by blue bands around the uterus. Areas selected for study in each uterus were cut open along the mesometrialantimesometrial plane with a sharp razor blade, the position and width of any blue bands were measured, and the tissue was corroded from the underlying vascular cast in $\mathrm{KOH}$ (approx 20\%, $w / v)$. Casts were washed, air dried, sputter-coated with gold and viewed in an ETEC-Siemens scanning electron microscope at $20 \mathrm{kV}$.

\section{Results}

Typical longitudinal sections of vascular casts from rats at Days 4, 5 and 6 of pregnancy are shown in Pl. 1, Figs 1-3.

Tissue staining from extravasation of Evans' blue was seen in one of the 4 uteri cast on Day 4, 5 of the 9 cast on Day 5 and all of the uteri cast on Day 6. In the Day 4 uterus that showed Evans' blue extravasation, the 6 bands were irregularly spaced and found only in the caudal half of the right horn. In the Day 5 uteri, one had faint signs of dye in some areas, 2 had irregularly spaced blue bands up to $10 \mathrm{~mm}$ wide, 1 had 13 discrete blue bands $1.0-2.5 \mathrm{~mm}$ wide and spaced $4.5-13.0 \mathrm{~mm}$ apart, and one was blue for its entire length, the blue fading slightly at the ovarian end of each horn but ending abruptly at the cervix. The finding of blue bands on Day 4 has not been reported before. The variation in uterine coloration on Day 5 was not related to the time of casting, because rats showing no blue colour at all were cast as late as $16: 30 \mathrm{~h}$, while the rat with 13 discrete blue bands was cast at $11: 50 \mathrm{~h}$.

A more consistent pattern was seen in the distribution of Evans' blue in the Day 6 specimens, with each rat having 5-8 blue bands in each uterine horn, the bands all being 2-4 mm in width and the spacing between bands in any horn being approximately even.

The appearance of the vascular casts from the 3 Day- 4 uteri without blue bands did not vary

\section{PLATE 1}

Longitudinal sections of vascular corrosion casts of the rat uterus during pregnancy. The casts were cut along the mesometrial-antimesometrial plane and the mesometrial border is at the top of each figure.

Fig. 1. Day 4 of pregnancy. Note the even spatial distribution of the endometrial subepithelial capillary plexus (SCP).

Fig. 2. Day 5 of pregnancy. Vertical lines indicate the extent of Evans' blue leakage around the implantation site (IS).

Fig. 3. Day 6 of pregnancy. Vertical lines indicate the extent of Evans' blue leakage around the implantation site (IS). Note the increased diameter and spacing of subepithelial capillaries around the implantation site, and the compaction of similar vessels in the non-blue regions. 

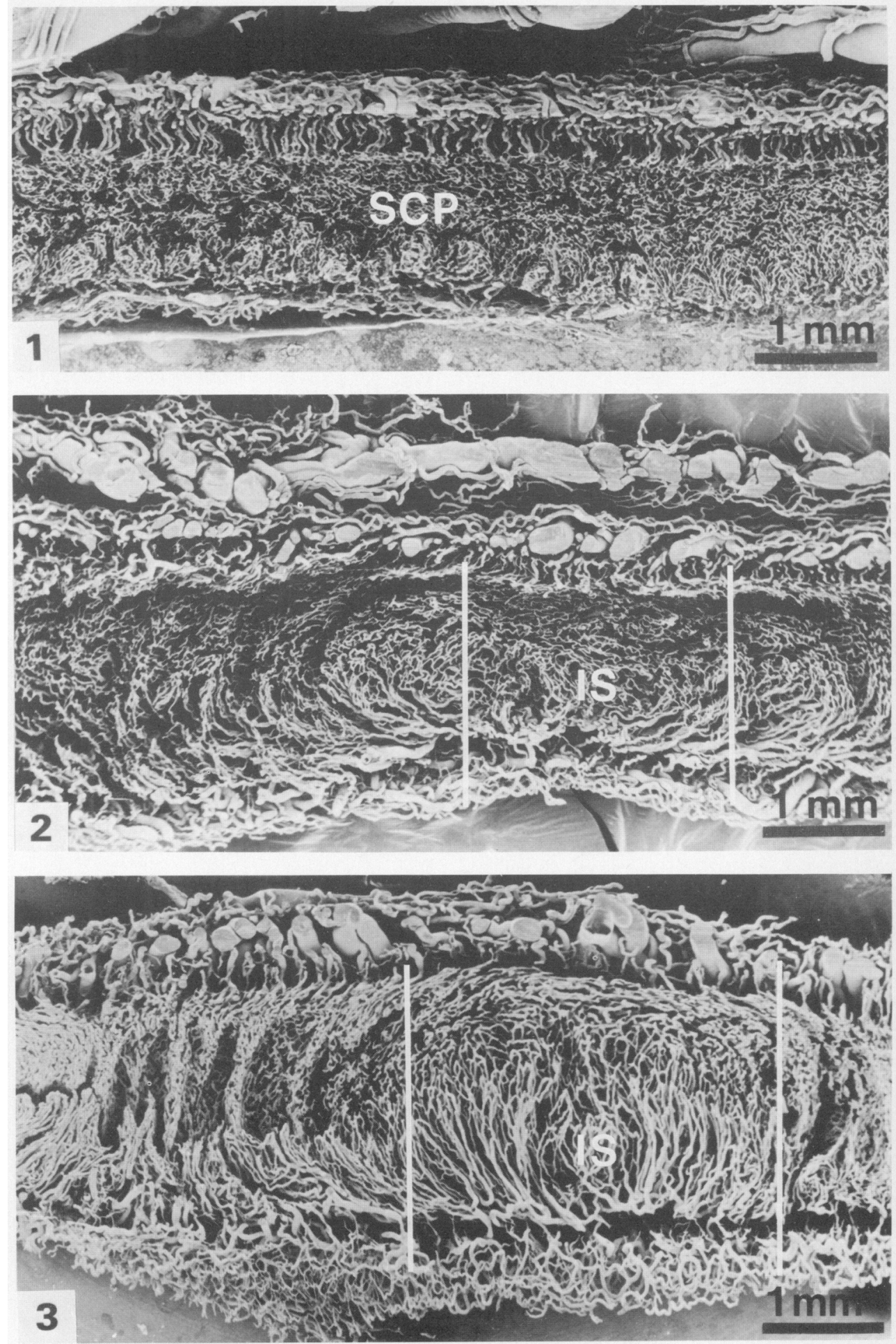

(Facing p. 212) 
along the length of the horns. The sub-epithelial capillary plexus was evenly distributed, and did not reflect any obvious folding of the endometrial tissues; at no point was there any apparent indication of where future implantation sites might be (see Pl. 1, Fig. 1). The Day 4 cast, at the blue band area, was similar in appearance to the Day 5 casts with blue banding, suggesting that this rat was 1 day earlier in the sequence of events leading to implantation than usual. Day 5 uterine casts from areas with no blue bands were essentially similar to those on Day 4 , although the luminal surface, as depicted by the sub-epithelial capillary plexus, tended to be more uneven, with an occasional transverse fold running through it. However, in areas where blue bands had been seen in the tissue, the Day 5 casts showed noticeable vascular changes (see Pl. 1, Fig. 2). The vessels of the sub-epithelial capillary plexus had begun to spread from a focal point, presumably the future implantation site. To either side of the blue band location the uterine vasculature appeared folded, as if the expansion of the tissues around the implantation site was causing compaction of tissues between sites. This expansion around the implantation site is most probably due to tissue hydration, caused by a local increase in vascular permeability at the implantation site, as demonstrated by the Evans' blue leakage.

By Day 6 the vascular cast of the uterus had taken on a striking appearance (see Pl. 1, Fig. 3 , with the vessels around each implantation site widely spaced, and the vessels in the areas between the sites being closely packed. At this stage of pregnancy, swelling of the uterus was visible at each implantation site, and this is reflected in the casts. Not only were all the vessels around the implantation site well spaced, but they appeared to be of much larger luminal diameter than vessels from the regions between sites or similar vessels on Days 4 or 5 .

\section{Discussion}

The present technique clearly shows the changes in uterine vascular architecture which accompany early implantation in the rat. By using the evenly spaced uterine blood vessels as positional markers (a 'vascular skeleton') in the uterine tissues before implantation, it is clear that implantation causes an increase in uterine length at each site, and a decrease in uterine length between sites. This is demonstrated by the increased spacing of blood vessels within the implantation site, and the decreased spacing of vessels between sites.

The localization of implantation sites by identifying surrounding areas of increased vascular permeability using an intravascular injection of Pontamine sky blue is a well accepted method (Finn \& Porter, 1975). In the present study, Evans' blue dye (mol. wt 960.8) was utilized; this dye binds strongly and rapidly to serum albumin after i.v. injection, (Freedman \& Johnson, 1969), and hence blue areas of the uterus indicate a local increase in vascular permeability to the extent that circulating proteins such as serum albumin (mol. wt 60000-70000) have leaked from the blood vessels into the surrounding tissues.

Differential growth of uterine tissues as a partial mechanism for blastocyst spacing has been postulated by a number of authors (see, for example, Reynolds, 1949; Perry \& Rowlands, 1962; Finn, 1968). However, the mechanism of this growth has not been demonstrated. Finn (1968) suggested that the increase in length of the uterus in the mouse at implantation may be due to endometrial hyperplasia around the implantation site. The present work demonstrates an increased spacing of the vascular skeleton in the areas where the Evans' blue-albumin complex leaked from the endometrial vessels. These areas will thus have a higher than normal interstitial oncotic pressure due to extravascular protein accumulation, and local Starling forces will thus favour local interstitial hydration and swelling. This type of response can occur very rapidly once an increase in vascular permeability is initiated, whereas a similar volume increase from cell division would take considerably longer. Therefore, the initial increase in uterine length is more probably due to local tissue hydration than to rapid cell division around the implantation sites. It is possible that this swelling initiates increased mitosis in surrounding tissues thus producing further uterine elongation at a later stage. 
In the present study, from cast measurements, the luminal diameters of sub-epithelial capillaries at the Day 6 implantation sites $(\simeq 20 \mu \mathrm{m})$ were larger than those in regions between implantation sites $(\simeq 14 \mu \mathrm{m})$. In the Day 5 cast, the sub-epithelial capillaries from areas of Evans' blue leakage did not, however, show this increase in vessel diameter over vessels from regions between sites (both $\simeq 8 \mu \mathrm{m}$ ). The luminal diameter of a blood vessel at the time of casting may not be exactly reproduced in the cast itself, due to a number of variables including a possible vasoactive effect of the casting medium. (For a full discussion on this matter see Rogers \& Gannon, 1981.) However, the reproducibility of this finding strongly suggests that the diameter changes seen in the casts reflect real changes in the vessels and that the initial vascular reaction at the implantation site is one of increasing permeability, with vaso-dilatation and presumably (although not necessarily) increased blood flow following later.

The variability in the timing of appearance of the Evans' blue leakage (as early as 11:30 h on Day 4, and no visible bands by 16:40 h on Day 5) has been reported by previous workers (see for example Tachi, Tachi \& Lindner, 1970), and is probably due to variations in the exact time of mating, ovulation and blastocyst arrival in the uterus from rat to rat.

In conclusion, this work, by the use of three-dimensional vascular corrosion casts made around the time of implantation, shows that differential uterine elongation occurs and could affect blastocyst spacing in the rat. The initial phase of this differential elongation appears to be due to tissue hydration following a local increase in endometrial microvascular permeability at the implantation sites.

We thank the Flinders University for a postgraduate scholarship (P.A.W.R.), the National Health and Medical Research Council for a grant to W. R. Jones and P.A.W.R. which partly supported the work and provided a fellowship (C.M.), and Miss J. Deer for typing the manuscript.

\section{References}

Boving, B.G. (1954) Blastocyst-uterine relationships. Cold Spring Harb. Symp. quant. Biol. 19, 9-25.

Finn, C.A. (1968) Increase in length of the uterus at the time of implantation in the mouse. J. Reprod. Fert. 17, 69-74.

Finn, C.A. \& Porter, D.G. (1975) Implantation of ova. In The Uterus, pp. 57-73. Elek Science, London.

Freedman, F.B. \& Johnson, J.A. (1969) Equilibrium and kinetic properties of the Evans' blue-albumin system. Am. J. Physiol. 216, 675-681.

Gannon, B.J. (1978) Vascular casting. In Principles and Techniques of Scanning Electron Microscopy, Vol. 6, pp. 170-193. Ed. M. A. Hayat. Van NostrandReinhold, New York.

Hammond, J. (1935) The changes in the reproductive organs of the rabbit during pregnancy. Trans. Dynamics of Growth 10, 93. (Cited by Reynolds, 1949.)

McLaren, A. \& Michie, D. (1959) The spacing of implantations in the mouse uterus. Mem. Soc. Endocr. 6, 65-75.

Mossman, H.W. (1937) Comparative morphogenesis of the fetal membranes and accessory uterine structures. Contr. Embryol. Carn. Instn 26, 129-246.

Murakami, T. (1971) Application of the scanning electron microscope to the study of the fine distribution of the blood vessels. Arch. Histol. Jap. 32, 445-454.

Perry, J.S. \& Rowlands, I.W. (1962) Early pregnancy in the pig. J. Reprod. Fert. 4, 175-188.

Pusey, J., Kelly, W.A., Bradshaw, J.M.C. \& Porter, D.G. (1980) Myometrial activity and the distribution of the blastocysts in the uterus of the rat: interference by relaxin. Biol. Reprod. 23, 394-397.

Reynolds, S.R.M. (1949) The Physiology of the Uterus. Hoeber, New York.

Rogers, P.A.W. Gannon, B.J. (1981) The vascular and microvascular anatomy of the rat uterus during the oestrous cycle. Aust. J. exp. Biol. Med. Sci. 59, $667-679$.

Tachi, S., Tachi, C. \& Lindner, H.R. (1970) Ultrastructural features of blastocyst attachment and trophoblastic invasion in the rat. J. Reprod. Fert. 21, $37-56$. 